\title{
Witnessing Multipartite Entanglement by Detecting Asymmetry
}

\author{
Davide Girolami *, ${ }^{\star}$ and Benjamin Yadin ${ }^{\dagger}$ \\ Department of Atomic and Laser Physics, University of Oxford, Parks Road, Oxford OX1 3PU, UK; \\ benjamin.yadin@wolfson.ox.ac.uk \\ * Correspondence: davegirolami@gmail.com \\ + These authors contributed equally to this work.
}

Academic Editor: Kevin H. Knuth

Received: 4 February 2017; Accepted: 12 March 2017; Published: 16 March 2017

\begin{abstract}
The characterization of quantum coherence in the context of quantum information theory and its interplay with quantum correlations is currently subject of intense study. Coherence in a Hamiltonian eigenbasis yields asymmetry, the ability of a quantum system to break a dynamical symmetry generated by the Hamiltonian. We here propose an experimental strategy to witness multipartite entanglement in many-body systems by evaluating the asymmetry with respect to an additive Hamiltonian. We test our scheme by simulating asymmetry and entanglement detection in a three-qubit Greenberger-Horne-Zeilinger (GHZ) diagonal state.
\end{abstract}

Keywords: quantum information; quantum coherence; entanglement; quantum metrology

\section{Introduction}

Quantum information theory provides important insights into the foundations of quantum mechanics, as well as its technological applications. The framework of resource theories characterizes the quantum laws as constraints, and the properties of quantum systems as resources for information processing [1]. In this context, the degree of coherent superposition of a state $\sum_{i} c_{i}|i\rangle\left\langle\left. i\left|, \sum_{i}\right| c_{i}\right|^{2}=1\right.$, i.e., coherence (we omit the quantum label, from now on) in a reference basis $\{i\}$, is a resource. The crucial question is to determine how to obtain a computational advantage powered by coherence [2-19]. The coherence of a finite-dimensional quantum state $\rho$ has been defined as its distinguishability from the sets of states which are diagonal in a given basis [14-19]. Yet, to date, there is no operational interpretation for such definition of coherence. A concurrent body of work has linked the coherence of $\rho$ in a basis $\{h\}$ to the degree of uncertainty in a measurement of an observable $H=\sum_{h} h|h\rangle\langle h|$ on $\rho$ [2-13]. Such genuinely quantum uncertainty is due to the non-commutativity between state and observable. Also, when the observable is a Hamiltonian generating a unitary evolution $e^{-i H t} \rho e^{i H t}$, the sensitivity of the state to the phase shift, which is called $U(1)$ - asymmetry, relies on coherence in the $H$ eigenbasis. The asymmetry of a quantum system quantifies its ability to break a symmetry generated by an observable $H$ (e.g., representing the energy). In particular, it measures the usefulness of the system as a reference frame under a superselection rule forbidding the preparation of coherent states in the $H$ eigenbasis [2]. Further studies bridged the gap between these recent theoretical findings and the experimental implementation of quantum information processing, by providing a strategy to measure the asymmetry of an arbitrary quantum state in the laboratory with the current technology [8] (for coherence witnesses, see [20-22]). These results paved the way for investigating the link between coherence and quantum properties of multipartite systems. In particular, the relationship between coherence and quantum correlations has been explored [6,9,13,16,18,19,23,24].

In this work, we show how detecting asymmetry in states of multipartite qubit systems allows an experimentalist to verify entanglement with limited resources. Entanglement is a crucial property 
for quantum information processing [25], e.g., providing speed-up in communication and metrology protocols [26,27]. Yet, it is hard to be quantified in both theoretical and experimental practice [28-30]. For this purpose, we here introduce an experimentally friendly witness of multipartite entanglement in terms of the asymmetry with respect to an additive Hamiltonian.

The structure of the paper is the following. In Section 2.1, we recall that the quantum Fisher information, a measure of sensitivity of a state to phase shifts employed in quantum metrology [27,31-33], is an asymmetry quantifier. It is possible to identify a lower bound of it in terms of traces of density matrix powers. We calculate how much the experimentally reconstructed bound deviates from the theoretical quantity (Section 2.2). Also, we express the lower bound for one, two and three-qubit states in terms of finite phase shifts generated by spin observables. These quantities can be evaluated by single qubit interferometry [34-38], as well as local projective measurement schemes [8,39-44], without performing full state reconstruction. In Section 3.1, we show that the asymmetry lower bound witnesses genuinely multipartite entanglement when measured with respect to an additive multipartite Hamiltonian. We complete the study with a demonstrative example (Section 3.2). We simulate the evaluation of asymmetry and entanglement in a Greenberger-Horne-Zeilinger (GHZ) diagonal state by a seven-qubit quantum information processor. We draw our conclusions in Section 4.

\section{Measuring Asymmetry}

\subsection{Theoretically Consistent Measure of Asymmetry}

In the resource theory of asymmetry [2-5,7], the consumable resource is any system whose state is not commuting with a fixed, bounded observable $H$ with spectral decomposition $H=\sum_{h} h|h\rangle\langle h|$. A system in the incoherent state $\rho_{H}=\sum_{h} c_{h}|h\rangle\langle h|,\left[\rho_{H}, H\right]=0$, is free, in the sense that it can be arbitrarily added or discarded in a quantum protocol without affecting the available asymmetry. Free states are invariant under phase rotations generated by $H: e^{-i H \theta} \rho_{H} e^{i H \theta}=\rho_{H}, \quad \forall \theta \in \mathbb{R}$. The free operations are the СРТP (completely-positive trace-preserving) maps $\mathcal{E}_{H}$ which cannot increase the amount of asymmetry in a state. That is, operations which cannot break the symmetry yielded by a Hamiltonian $H$. They are identified by maps commuting with the unitary evolutions generated by the observable under scrutiny, $e^{-i H \theta} \mathcal{E}_{H}(\rho) e^{i H \theta}=\mathcal{E}_{H}\left(e^{-i H \theta} \rho e^{i H \theta}\right), \quad \forall \rho, \theta$. Their explicit form in terms of Kraus operators has been identified [3]. Several quantifiers of asymmetry have been proposed $[3,7,8]$. Here, we adopt the viewpoint of asymmetry as a measure of the state usefulness in a phase estimation scenario. The symmetric logarithmic derivative quantum Fisher information is indeed a measure of asymmetry [7,33]. Let us recall its definition. Given the spectral decomposition of a probe state $\rho=\sum_{i} \lambda_{i}|i\rangle\langle i|, \sum_{i} \lambda_{i}=1$, and an observable $H$, the quantum Fisher information $\mathcal{F}_{H}(\rho)=$ $2 \sum_{i, j} \frac{\left(\lambda_{i}-\lambda_{j}\right)^{2}}{\lambda_{i}+\lambda_{j}} H_{i j}^{2}, H_{i j}=|\langle i|H| j\rangle|$, quantifies the sensitivity of the probe to a phase shift $U_{H}(\theta)=e^{-i H \theta}$ generated by $H$, under the assumption that the state changes smoothly [31]. The quantum Fisher information is (four times) the convex roof of the variance, $\mathcal{V}_{H}(|\psi\rangle):=4\left(\left\langle\psi\left|H^{2}\right| \psi\right\rangle-\langle\psi|H| \psi\rangle^{2}\right)$, meaning that $\mathcal{F}_{H}(\rho)=\inf _{\left\{p_{i}\left|\psi_{i}\right\rangle\right\}} \sum_{i} p_{i} \mathcal{V}_{H}\left(\left|\psi_{i}\right\rangle\right)$, where the infimum is taken over all the convex decompositions $\rho=\sum_{i} p_{i}\left|\psi_{i}\right\rangle\left\langle\psi_{i}\right|$ such that the $\left\{p_{i}\right\}$ form a probability distribution $[45,46]$. Moreover, a decomposition saturating the equality always exists. This property implies convexity, $\mathcal{F}_{H}(p \rho+(1-p) \sigma) \leq p \mathcal{F}_{H}(\rho)+(1-p) \mathcal{F}_{H}(\sigma)$. The quantum Fisher information is equal to the variance for pure states, $\mathcal{F}_{H}(|\psi\rangle\langle\psi|)=\mathcal{V}_{H}(|\psi\rangle)$.

We recall what implies that the quantum Fisher information is a reliable measure of asymmetry. It satisfies the following criteria:

(i) It vanishes if and only if the state is incoherent. Since the quantum Fisher information is convex, for any incoherent state, one has $\mathcal{F}_{H}\left(\rho_{H}\right)=\mathcal{F}_{H}\left(\sum_{h} c_{h}|h\rangle\langle h|\right) \leq \sum_{h} c_{h} \mathcal{F}_{H}(|h\rangle\langle h|)=0$. Also, we observe that $\mathcal{F}_{H}(\rho)=0 \Leftrightarrow[\rho, H]=0$, and $[\rho, H]=0 \Leftrightarrow\left[\rho, U_{H}(\theta)\right]=0, \forall \theta$, which is a condition satisfied if and only if the state is incoherent. 
(ii) It cannot increase under free operations. Given $H_{A B}=H_{A} \otimes I_{B}+I_{A} \otimes H_{B}$, by Theorem II.1 of Ref. [5], any map $\mathcal{E}_{H_{A}}$ admits a Stinespring dilation $\mathcal{E}_{H_{A}}\left(\rho_{A}\right)=\operatorname{Tr}_{B}\left[V_{A B}^{H}\left(\rho_{A} \otimes \tau_{B}\right) V_{A B}^{H+}\right]$, where $V_{A B}^{H}$ is a free unitary with respect to $H_{A B}$, and $\left[\tau_{B}, H_{B}\right]=0$. In other words, any free map can be represented by the unitary, free evolution of the system of interest and an ancilla in an incoherent state (i.e., a free state). One then obtains $\mathcal{F}_{H_{A}}\left(\rho_{A}\right)=\mathcal{F}_{H_{A B}}\left(\rho_{A} \otimes \tau_{B}\right)=$ $\mathcal{F}_{H_{A B}}\left(V_{A B}^{H+}\left(\rho_{A} \otimes \tau_{B}\right) V_{A B}^{H}\right) \geq \mathcal{F}_{H_{A}}\left(\operatorname{Tr}_{B}\left[V_{A B}^{H+}\left(\rho_{A} \otimes \tau_{B}\right) V_{A B}^{H}\right]\right)=\mathcal{F}_{H_{A}}\left(\mathcal{E}_{H_{A}}\left(\rho_{A}\right)\right)$. The first equality is due to the additivity of the quantum Fisher information for additive observables and uncorrelated states. The second one is due to the invariance of the quantum Fisher information under unitaries applied to both the state and the Hamiltonian, and to the fact that the unitary here is a free operation, $\left[V_{A B}^{H}, H_{A B}\right]=0$. The inequality is due to the contractivity of the quantum Fisher information under partial trace.

The proof can be extended to any quantum Fisher information $\mathcal{I}_{H}^{f}(\rho)=\sum_{i, j} \frac{\left(\lambda_{i}-\lambda_{j}\right)^{2}}{\lambda_{j} f\left(\lambda_{i} / \lambda_{j}\right)}|\langle i|H| j\rangle|^{2}$, where each of the real-valued functions $f$ identifies a quantization of the classical Fisher information which preserves contractivity under noisy operations, being $\mathcal{F}_{H}(\rho)=\mathcal{I}_{H}^{F}(\rho), F(x)=(1+x) / 2$, $x \in \mathbb{R}$ [47]. The quantum Fisher information is topologically equivalent, being connected by the chain $2 f(0) \mathcal{I}_{H}^{f}(\rho) \leq \mathcal{F}_{H}(\rho) \leq \mathcal{I}_{H}^{f}(\rho), \forall f, \rho, H$ [48]. Also, the property ii) can be generalized to show that any quantum Fisher information is an ensemble monotone, i.e., it does not increase on average under free operations, $\mathcal{I}_{H}^{f}(\rho) \geq \sum_{\mu} p_{\mu} \mathcal{I}_{H}^{f}\left(\mathcal{E}_{\mu}(\rho)\right), \forall\left\{p_{\mu}, \mathcal{E}_{\mu}\right\}: \sum_{\mu} p_{\mu}=1,\left[\mathcal{E}_{\mu}, U_{H}(\theta)\right]=0, \forall f[33]$

Picking the Fisher information as a measure of asymmetry is useful for experimental purposes. Coherence is not a linear property of a system, so it cannot be directly related to a quantum operator [49]. Also, the quantum Fisher information is usually hard to compute. Yet, it is possible to build up an observable quantity which provides a nontrivial lower bound:

$$
\begin{aligned}
& \mathcal{O}_{H}(\rho) \leq \mathcal{F}_{H}(\rho), \\
& \mathcal{O}_{H}(\rho)=-2 \operatorname{Tr}\left[[\rho, H]^{2}\right]=4 \operatorname{Tr}\left[\rho^{2} H^{2}-\rho H \rho H\right] .
\end{aligned}
$$

As previously observed [33], one has $\mathcal{O}_{H}(\rho)=2 \sum_{i \neq j}\left(\lambda_{i}-\lambda_{j}\right)^{2} H_{i j}^{2}$. Since $\lambda_{i}+\lambda_{j} \leq 1, \forall i, j$, by recalling the expression of the quantum Fisher information, the lower bound holds. For pure states, one has $\mathcal{O}_{H}(\rho)=\mathcal{F}_{H}(\rho)=4 \mathcal{V}_{H}(\rho)$. The lower bound reliably detects asymmetry, as $\mathcal{O}_{H}(\rho)=0 \Leftrightarrow$ $\mathcal{F}_{H}(\rho)=0$.

One may wonder if the quantity $\mathcal{O}_{H}(\rho)$ itself is a consistent measure of asymmetry. For pure states, the lower bound equals the quantum Fisher information, so the answer is positive in such a case. Unfortunately, this does not hold for mixed states. We can see this with a simple example. Given a bipartite state $\rho_{A B}=\rho_{A} \otimes \rho_{B}$, let us suppose that the asymmetry of the marginal state is measured $\rho_{A}$ as the uncertainty measuring $H_{A}$. One obtains $\mathcal{O}_{H_{A}}\left(\rho_{A B}\right)=\mathcal{O}_{H_{A}}\left(\rho_{A}\right) \operatorname{Tr}\left[\rho_{B}^{2}\right]$. Then, discarding the subsystem $\rho_{B}$ would increase the asymmetry of the state $\rho_{A}$, which is manifestly undesirable. One may normalize the quantity by employing $\mathcal{O}_{H}(\rho) / \operatorname{Tr}\left[\rho^{2}\right]$ as a measure of asymmetry, yet there would still be a problem. Note that the bound is written (modulo a constant) as an Hilbert-Schmidt norm in the zero shift limit, $\mathcal{O}_{H}(\rho)=2 \lim _{\theta \rightarrow 0}\left\|U_{H}(\theta) \rho U_{H}(\theta)^{\dagger}-\rho\right\|_{2}^{2} /\left(\theta^{2}\right)$. This norm is notoriously not contractive under quantum operations [50]. Not surprisingly, this property also makes measures of quantum correlations based on this norm generally unreliable [51,52]. Hence, the lower bound, while being not a full-fledged measure, can replace the quantum Fisher information in scenarios where some restriction is posed, e.g., for unitary evolutions of systems which are guaranteed to be closed.

\subsection{Experimental Observability of the Asymmetry Bound}

It has been proven that the asymmetry lower bound is a function of mean values of self-adjoint operators [8]. By applying the Taylor expansion about $\theta=\theta_{0}$, one has $\operatorname{Tr}\left[\rho U_{H}(\theta) \rho U_{H}^{+}(\theta)\right]=\operatorname{Tr}\left[\rho U_{H}\left(\theta_{0}\right) \rho U_{H}^{+}\left(\theta_{0}\right)\right]-\left(\operatorname{Tr}\left[\rho^{2} H^{2}\right]-\operatorname{Tr}[\rho H \rho H]\right)\left(\theta-\theta_{0}\right)^{2}+O\left(\left(\theta-\theta_{0}\right)^{3}\right)$, and 
then $\mathcal{O}_{H}(\rho) \sim \frac{4}{\left(\theta-\theta_{0}\right)^{2}}\left(\operatorname{Tr}\left[\rho U_{H}\left(\theta_{0}\right) \rho U_{H}^{\dagger}\left(\theta_{0}\right)\right]-\operatorname{Tr}\left[\rho U_{H}(\theta) \rho U_{H}^{+}(\theta)\right]\right), \theta \rightarrow \theta_{0}$. By setting $\theta_{0}=0$, an approximation in terms of finite phase shifts, with error $O\left(\theta^{2}\right)$, is given by $\mathcal{O}_{H}^{\text {ap }}(\rho) \pm \Delta \mathcal{O}_{H}^{\text {ap }}(\rho)$, with

$$
\begin{aligned}
\mathcal{O}_{H}^{\mathrm{ap}}(\rho) & =4 \frac{\operatorname{Tr}\left[\rho^{2}\right]-\operatorname{Tr}\left[\rho U_{H}(\theta) \rho U_{H}^{+}(\theta)\right]}{\theta^{2}} \\
\Delta \mathcal{O}_{H}^{\mathrm{ap}}(\rho) & =\underbrace{\left|d \mathcal{O}_{H}^{\mathrm{ap}}(\rho) / d \theta\right|_{\theta=0}}_{=0} \theta+1 / 2\left|d^{2} \mathcal{O}_{H}^{\mathrm{ap}}(\rho) / d \theta^{2}\right|_{\theta=0} \theta^{2} .
\end{aligned}
$$

One may note that even the approximated quantity is a lower bound (but less tight) to the quantum Fisher information, $\mathcal{O}_{H}^{\mathrm{ap}}(\rho) \leq \mathcal{O}_{H}(\rho), \forall \rho, H, \theta$ [33]. Therefore, to quantify the lower bound to the asymmetry of the state, we need to evaluate its purity and the overlap with a second copy of the state after a rotation has been applied. They are obtained by estimating the mean value of the swap operator $V=\sum_{i j}|i j\rangle\langle j i|$ in two copies of the system, $\rho_{1,2} \equiv \rho: \operatorname{Tr}\left[\rho^{2}\right]=\operatorname{Tr}[V(\rho \otimes \rho)]$, while the overlap is given by $\operatorname{Tr}\left[\rho U_{H} \rho U_{H}^{+}\right]=\operatorname{Tr}\left[V\left(\rho \otimes U_{H} \rho U_{H}^{+}\right)\right]$. Such quantities can be directly measured by implementing an interferometric configuration $[8,34-39,53]$. In fact, the method has general validity regardless the system state and the self-adjoint operator to be measured [34]. Alternatively, for the relevant case of $\mathrm{N}$-qubit systems, it is possible to extract purity and overlap by local Bell measurements, a routine measurement scheme in optical setups $[8,33,39-44]$. Thus, for systems of arbitrary dimension, the lower bound $O_{H}(\rho)$ can be extracted by the statistics of a limited number of detections, bypassing full state reconstruction.

It is possible to derive a closed formula for the asymmetry lower bound in one, two and three-qubit states, with respect to additive Hamiltonians $H_{N}=\sum_{i=1}^{N} h_{i}, h_{i}=I_{1,2, \ldots, i-1} \otimes h_{i} \otimes I_{i+1, i+2, \ldots, N}, h_{i}=$ $1 / 2 \sigma_{i}, \sigma_{i}$ representing spin-1/2 observables, e.g., the Pauli matrices. By recalling that $e^{i \sigma \theta / 2}=$ $\cos \theta / 2 I_{2}+i \sin \theta / 2 \sigma$, we get an exact expression for the lower bound in terms of phase shifts $U_{H_{N}}(\theta)=e^{-i H_{N} \theta}$. For $N=1, H_{1}=h$, one has

$$
\mathcal{O}_{H_{1}}(\rho)=\operatorname{Tr}\left[\rho^{2}\right]-\operatorname{Tr}\left[\rho U_{h}(\pi) \rho U_{h}^{\dagger}(\pi)\right],
$$

For $N=2, H_{2}=h_{1}+h_{2}$ :

$$
\begin{aligned}
\mathcal{O}_{H_{2}}(\rho) & =3 \operatorname{Tr}\left[\rho^{2}\right]-4 \operatorname{Tr}\left[\rho U_{H_{2}}(\pi / 2) \rho U_{H_{2}}^{\dagger}(\pi / 2)\right] \\
& +\operatorname{Tr}\left[\rho U_{H_{2}}(\pi) \rho U_{H_{2}}^{+}(\pi)\right], \\
U_{H_{2}}(\theta) & =U_{h_{1}}(\theta) U_{h_{2}}(\theta) .
\end{aligned}
$$

For $N=3, H_{3}=h_{1}+h_{2}+h_{3}$ :

$$
\begin{aligned}
\mathcal{O}_{H_{3}}(\rho) & =6 \operatorname{Tr}\left[\rho^{2}\right]-4\left\{\operatorname{Tr}\left[\rho U_{h_{1}+h_{2}}(\pi / 2) \rho U_{h_{1}+h_{2}}^{+}(\pi / 2)\right]\right. \\
& +\operatorname{Tr}\left[\rho U_{h_{1}+h_{3}}(\pi / 2) \rho U_{h_{1}+h_{3}}^{+}(\pi / 2)\right] \\
& \left.+\operatorname{Tr}\left[\rho U_{h_{2}+h_{3}}(\pi / 2) \rho U_{h_{2}+h_{3}}^{+}(\pi / 2)\right]\right\} \\
& +\operatorname{Tr}\left[\rho U_{h_{1}+h_{2}}(\pi) \rho U_{h_{1}+h_{2}}^{+}(\pi)\right]+\operatorname{Tr}\left[\rho U_{h_{1}+h_{3}}(\pi) \rho U_{h_{1}+h_{3}}^{+}(\pi)\right] \\
& +\operatorname{Tr}\left[\rho U_{h_{2}+h_{3}}(\pi) \rho U_{h_{2}+h_{3}}^{+}(\pi)\right] \\
& +\operatorname{Tr}\left[\rho U_{h_{1}}(\pi) \rho U_{h_{1}}^{+}(\pi)\right]+\operatorname{Tr}\left[\rho U_{h_{2}}(\pi) \rho U_{h_{2}}^{\dagger}(\pi)\right] \\
& +\operatorname{Tr}\left[\rho U_{h_{3}}(\pi) \rho U_{h_{3}}^{+}(\pi)\right] .
\end{aligned}
$$

We conjecture that it is possible to iterate the procedure and work out equivalent expressions for an arbitrary number of qubits. 


\section{Detection of Multipartite Entanglement via Asymmetry}

\subsection{Asymmetry Witnesses Entanglement}

It is often desirable to consider a high dimensional system as a partition of subsystems. Such a partition is usually dictated by the physical constraints of the problem, for example the spatial separation between the parts of the system. It is then interesting to understand the interplay between asymmetry with respect to a global observable and the quantum properties of the subsystems. In spite of being a basis-dependent feature, coherence is linked to basis-independent features of multipartite systems as quantum correlations $[6,16,18,23,28]$. Here we show that, for an $N$-qubit system, the observable asymmetry bound $\mathcal{O}_{H}(\rho)$ measured on the global system state witnesses entanglement between the partitions. There are several entanglement witnesses written in terms of the quantum Fisher information. They relate entanglement to the system speed of response to phase shifts generated by additive spin-1/2 Hamiltonians $J_{N}=\sum_{i=1}^{N} 1 / 2 \sigma_{i}$ [32,46,54-60]. In particular, a constraint which cannot be satisfied by $k$-separable states of $N$ qubits is $\mathcal{F}_{J_{N}}(\rho) \geq n k^{2}+(N-n k)^{2}$, where $n=\left\lfloor\frac{N}{k}\right\rfloor$. Thus, verifying this relation certifies genuine $k$-partite entanglement [25]. Also, if $\overline{\mathcal{F}}(\rho)=1 / 3\left(\mathcal{F}_{J_{N, x}}(\rho)+\mathcal{F}_{J_{N, y}}(\rho)+\mathcal{F}_{J_{N, z}}(\rho)\right)>2 N / 3$, then the state is entangled. Therefore, a state $\rho$ is genuinely $k$-partite entangled if there exists a spin basis $\{x, y, z\}$ such that:

$$
\begin{aligned}
\mathcal{O}_{J_{N, x(y, z)}}(\rho) & >n k^{2}+(N-n k)^{2} \\
\overline{\mathcal{O}}(\rho) & =1 / 3\left(\mathcal{O}_{J_{N, x}}(\rho)+\mathcal{O}_{J_{N, y}}(\rho)+\mathcal{O}_{J_{N, z}}(\rho)\right)>2 N / 3 .
\end{aligned}
$$

Table 1. Theoretical values of the quantum Fisher information, the observable lower bound defined in Equation (1), and the conditions witnessing entanglement, Equation (6), given the spin observables $J_{N, x(y, z)}$, for $k=1,2$, in $\rho_{G H Z(D)}^{p}$. For mixtures of $N$ qubit pure states ( $N$ even) and white noise, $\rho_{\psi}^{p}=(1-p) / 2^{N} I+$ $p|\psi\rangle\langle\psi|$, one has $\mathcal{F}_{H}\left(\rho_{\psi}^{p}\right)=4 p^{2} /\left(p+(1-p) /\left(2^{N+1}\right)\right) \mathcal{V}_{H}(|\psi\rangle)$ [32], and $\mathcal{O}_{H}\left(\rho_{\psi}^{p}\right)=4 p^{2} \mathcal{V}_{H}(|\psi\rangle), \forall H$. The implicit functions in the table read $f_{1}(N)=\frac{2^{-N-2}\left(2^{N+1}-1\right)+\frac{1}{4} \sqrt{2^{-2 N}\left(2^{N+3} N-2^{N+2}+2^{2 N+2}+1\right)}}{N}, f_{2}(N)=$ $\frac{2^{-N-1}\left(2^{N+1}-1\right)+\frac{1}{2} \sqrt{2^{-2 N}\left(2^{N+2} N-2^{N+2}+2^{2 N+2}+1\right)}}{N}, g_{1}(N)=\frac{2^{-N-1}\left(2^{N+1}-1\right)+\frac{1}{2} \sqrt{2^{-2 N}\left(2^{N+2} N+2^{N+2}+2^{2 N+2}+1\right)}}{N+2}$ and $g_{2}(N)=\frac{2^{-N-1}\left(2^{N+1}-1\right)+\sqrt{2^{-2 N}\left(2^{N+1} N+2^{2 N+2}+1\right)}}{N+2}$. It is not always possible to obtain a meaningful entanglement witness: we use the label / whenever the witnessing condition is satisfied for unphysical

\begin{tabular}{|c|c|c|c|}
\hline $\begin{array}{ll}\mathrm{J}_{\mathbf{N}} & \mathrm{J}_{\mathbf{N}}\end{array}$ & $\mathbf{J}_{\mathbf{N}, \mathbf{x}}$ & $\mathrm{J}_{\mathbf{N}, \mathbf{y}}$ & $\mathbf{J}_{\mathbf{N}, \mathbf{z}}$ \\
\hline $\mathcal{F}_{J_{N}}\left(\rho_{G H Z}^{p}\right)$ & $p>f_{1}(N), \quad p>f_{2}(N)$ & I & / \\
\hline $\mathcal{O}_{J_{N}}\left(\rho_{G H Z}^{p}\right)$ & $p>1 / \sqrt{N}, p>\sqrt{2 / N}$ & / & / \\
\hline $\mathcal{F}_{J_{N}}\left(\rho_{D}^{p}\right)$ & $p>g_{1}(N), \quad p>g_{2}(N)$ & $p>g_{1}(N), \quad p>g_{2}(N)$ & 1 \\
\hline $\mathcal{O}_{J_{N}}\left(\rho_{D}^{p}\right)$ & $p>\sqrt{\frac{2}{(N+2)}}, p>\frac{2}{\sqrt{N+2}}$ & $p>\sqrt{\frac{2}{(N+2)}}, p>\frac{2}{\sqrt{N+2}}$ & / \\
\hline
\end{tabular}
values $p \notin[0,1]$.

We compute the first entanglement witness condition for highly entangled states of $N$ qubits (assuming $N$ even) employed in quantum metrology, the generalized GHZ and the $N / 2$ excitation Dicke states, mixed with white noise, $\rho_{G H Z(D)}^{p}=(1-p) / 2 \mathbb{I}+p|G H Z(D)\rangle\langle G H Z(D)|| G H Z\rangle=$, $1 / \sqrt{2}\left(|0\rangle^{\otimes N}+|1\rangle^{\otimes N}\right),|D\rangle=\frac{1}{\sqrt{\left({ }_{N / 2}^{N}\right)}} \sum_{i} P_{i}\left(|0\rangle^{\otimes(N / 2)} \otimes|1\rangle^{\otimes(N / 2)}\right), p \in[0,1]$, where $P_{i}$ are the subsystem permutations [32]. The results are given in Table 1. An interesting alternative option is to build entanglement witnesses in terms of the average values and the variances of the collective spin operators (see [32] and references therein, and a newer proposal in [61]). The advantage of such methods is that the witness can be calculated without actually performing the phase shift, just by measuring the spin values on the probe state. We note that such witnesses, including ours, determine metrological useful entanglement yielding super-linear scaling of lower bounds of the quantum Fisher 
information, rather than just non-separability of the quantum state. The choice of the appropriate witness depends on the particulars of the experimental setting, e.g., the a priori knowledge of the probe state, the probe state itself, the chosen measurement strategy (for example, collective measurements rather than single-site detections).

\subsection{A Case Study}

Here we apply our scheme to simulate the non-tomographic detection of asymmetry and entanglement in a three-qubit state. We choose the GHZ-diagonal state $\rho_{\mathrm{ABC}}^{p}$ as probe state. This allows one to investigate the behavior of the asymmetry lower bound and entanglement witness in the presence of noise in the system. The two copies of the GHZ diagonal state $\rho_{A_{1} B_{1} C_{1}}^{p} \rho_{A_{2} B_{2} C_{2}}^{p}$ are obtained by initializing a six qubit processor in $\rho_{i}=1 / 2\left(I_{2}+p \sigma_{z}\right), i=i \ldots, 6$, and applying Hadamard and controlled-not (CNOT) gates as described in Figure 1.

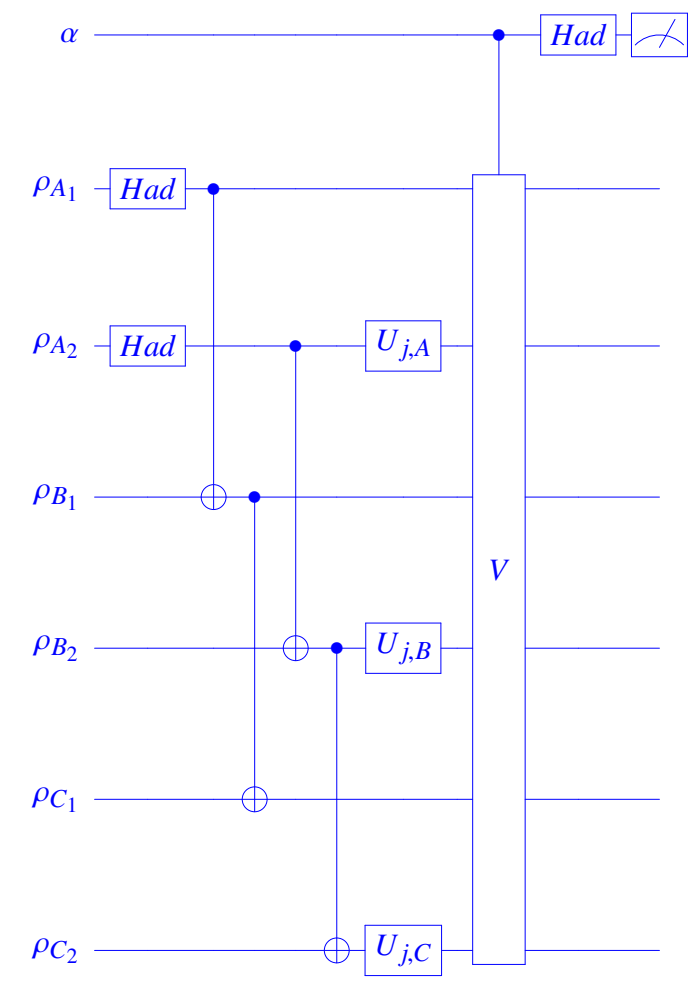

Figure 1. Overlap detection. Two copies $\rho_{A_{1} B_{1} C_{1}}^{p}, \rho_{A_{2} B_{2} C_{2}}^{p}$ of a GHZ-diagonal state are prepared in the state $\rho_{i}=1 / 2\left(I_{2}+p \sigma_{z}\right), \forall i$. An Hadamard gate $\mathrm{Had}$ is applied to the qubits $A_{i}, i=1,2$, followed by two CNOT gates on each copy. Then, one evaluates the purity and the overlap terms related to the observables $J_{3, x(y, z)}$, by applying the unitary transformations $U_{J_{3}}(\theta)=U_{j, A}(\theta) \otimes U_{j, B}(\theta) \otimes U_{j, C}(\theta)$, and measuring the ancilla polarisation by means of an interferometric scheme. This consists of an ancilla in the initial state $\alpha=1 / \sqrt{2}(|0\rangle+|1\rangle)$ interacting with the two state copies by a controlled- $V$ gate, being $V$ the swap operator. A second Hadamard gate $H$ is finally applied to the ancilla. The mean value of the ancilla polarisation at the output is $\left\langle\sigma_{z}\right\rangle_{\alpha^{\text {out }}}=\operatorname{Tr}\left[V \rho \otimes U_{J_{3}}(\theta) \rho U_{J_{3}}^{\dagger}(\theta)\right]=\operatorname{Tr}\left[\rho U_{J_{3}}(\theta) \rho U_{J_{3}}^{\dagger}(\theta)\right]$, which determines the asymmetry lower bound.

We measure the asymmetry of the input state with respect to the set of spin Hamiltonians $J_{3}=\sum_{i=A, B, C} j_{3, i}, j_{3, A}=j_{A} \otimes I_{B C}, j_{3, B}=I_{A} \otimes j_{B} \otimes I_{C}, j_{3, C}=I_{A B} \otimes j_{C}, j=1 / 2 \sigma_{x,(y, z)}$, by computing the values of the lower bound, and the approximation defined in Equation (2), for each observable. Of course, we may obtain the asymmetry with respect to any self-adjoint operator in the three-qubit Hilbert space. This is done by implementing the unitary gate $U_{J_{3}}(\theta)=U_{j, A}(\theta) \otimes U_{j, B}(\theta) \otimes U_{j, C}(\theta)$ on a copy of the state and then building up an interferometric configuration (Figure 1). Performing 
the polarisation measurements on the ancillary qubit makes it possible to determine $\mathcal{O}_{J_{3}}\left(\rho_{A B C}^{p}\right)$. We select a small but experimentally plausible phase shift, $\theta=\pi / 6$ [33]. Obviously, to evaluate the purity, no gate has to be applied. The purity and overlap values extracted by the quantities $\operatorname{Tr}\left[\rho_{A B C}^{p} U_{J_{3}}(\pi / 6) \rho_{A B C}^{p} U_{J_{3}}^{\dagger}(\pi / 6)\right]$ determine $\mathcal{O}_{I_{3}}^{\text {ap }}\left(\rho_{A B C}^{p}\right)$. No further action is necessary to verify the presence of entanglement through the witnesses in Equation (6), as the values of $\mathcal{O}_{J_{3}}^{\mathrm{ap}}\left(\rho_{A B C}^{p}\right)$ have been obtained in the previous steps. For $N=3$, we have $k=1 \Rightarrow \mathcal{O}_{J_{3}}\left(\rho_{A B C}^{p}\right) \geq 3, k=2 \Rightarrow \mathcal{O}_{J_{3}}\left(\rho_{A B C}^{p}\right) \geq 5$, and $\overline{\mathcal{O}}\left(\rho_{A B C}^{p}\right)>2$. The results are summarised in Table 2 and Figures 2 and 3.

Table 2. Theoretical values of the quantum Fisher information, the observable lower bound defined in Equation (1), and the conditions witnessing entanglement, Equation (6), for the spin observables $J_{3, x(y, z)}$, in $\rho_{A B C}^{p}$. The coherence lower bound is an entanglement witness that is almost as efficient as the quantum Fisher information, being blind to entanglement only for $p \in[0.674,0.751],[0.646,0.772]$, and to tripartite entanglement for $p \in[0.813,0.861]$. Note that a more general sufficient condition for genuine tripartite entanglement is $\left|\rho_{1,8}\right|>\sqrt{\rho_{2,2} \rho_{7,7}}+\sqrt{\rho_{3,3} \rho_{6,6}}+\sqrt{\rho_{4,4} \rho_{5,5}}$, which for GHZ-diagonal states is also a necessary condition [60]. Hence, $\rho_{A B C}^{p}$ is three-partite entangled when $p>2^{2 / 3}-1 \approx 0.587$.

\begin{tabular}{c|ccc}
\hline $\mathbf{J}_{3}$ & $\mathbf{J}_{3, \mathbf{x}}$ & $\mathbf{J}_{3, \mathbf{y}}$ & $\mathbf{J}_{3, \mathbf{z}}$ \\
\hline $\mathcal{F}_{J_{3}}\left(\rho_{A B C}^{p}\right)$ & $\frac{2 p^{2}\left(p^{2}+2\right)}{p^{2}+1}$ & $\frac{\left(-2 p^{8}+p^{6}+18 p^{4}+7 p^{2}\right)}{3 p^{4}+4 p^{2}+1}$ & $\left(2 p^{4}+4 p^{3}+3 p^{2}\right)$ \\
\hline $\mathcal{O}_{J_{3}}\left(\rho_{A B C}^{p}\right)$ & $\left(2 p^{2}+3 p^{4}+p^{6}\right) / 2$ & $\left(p^{6}+4 p^{4}+7 p^{2}\right) / 4$ & $\left(3 p^{6}+8 p^{5}+14 p^{4}+8 p^{3}+3 p^{2}\right) / 4$ \\
\hline $\mathcal{F}_{J_{3}}\left(\rho_{A B C}^{p}\right)>3,5$ & $/$ & $/$ & $p>0.674,0.813$ \\
\hline $\mathcal{O}_{J_{3}}\left(\rho_{A B C}^{p}\right)>3,5$ & $/$ & $/$ & $p>0.751,0.861$ \\
\hline$\overline{\mathcal{F}}\left(\rho_{A B C}^{p}\right)>2, \overline{\mathcal{O}}\left(\rho_{A B C}^{p}\right)>2$ & \multicolumn{4}{|c}{$p>0.646,0.772$} \\
\hline
\end{tabular}

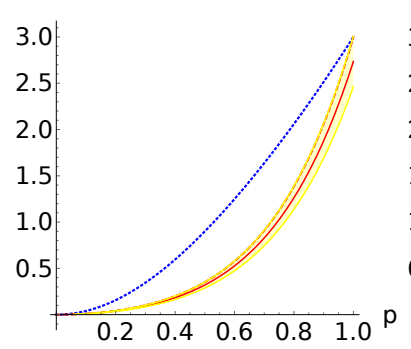

(a)

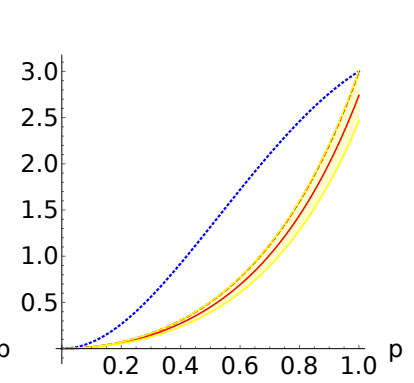

(b)

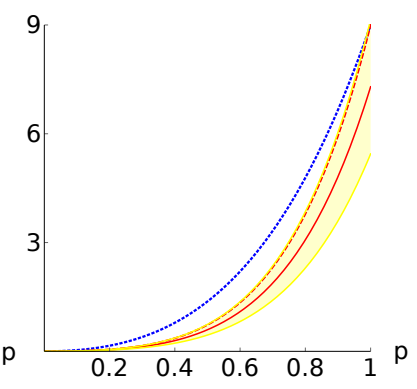

(c)

Figure 2. (Colors Online)—Evaluation of asymmetry in the state $\rho_{A B C}^{p}$ with respect to the observables $J_{3, x(y, z)}$ (figures (a)-(c) respectively) as a function of the mixing parameter $p$. The blue dotted line is the quantum Fisher information, here shown for reference; the red dashed line is the bound $O_{J_{3}}\left(\rho_{A B C}^{p}\right)$; the red continuous line is the approximation $O_{J_{3}}^{\mathrm{ap}}\left(\rho_{A B C}^{p}\right)$ obtained by imposing $\theta=\pi / 6$; and the yellow band is the error region, whose extreme values are $O_{J_{3}}^{\mathrm{ap}}\left(\rho_{A B C}^{p}\right) \pm \Delta O_{J_{3}}^{\mathrm{ap}}\left(\rho_{A B C}^{p}\right)$. 


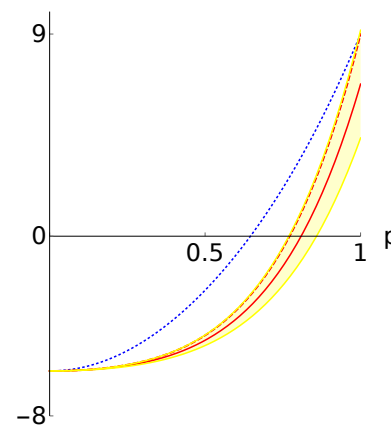

(a)

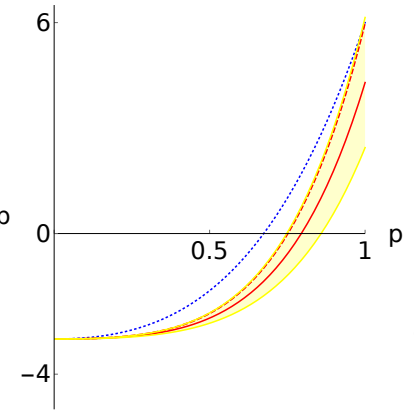

(b)

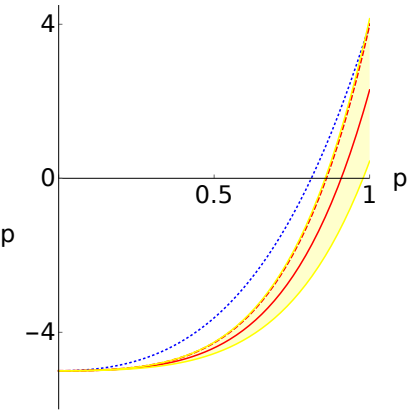

(c)

Figure 3. (Colors Online)-Witnessing entanglement by asymmetry via the inequalities in Equation (6). (a) Witnessing entanglement in the state $\rho_{A B C}^{p}$ by computing the quantum Fisher information and the lower bound, as a function of the mixing parameter $p$. The blue dotted line depicts $\mathcal{F}_{J_{z, 3}}\left(\rho_{A B C}^{p}\right)-3$, the red dashed line is $\mathcal{O}_{J_{3, z}}\left(\rho_{A B C}^{p}\right)-3$, while the red continuous line is $O_{J_{3}}^{\text {ap }}\left(\rho_{A B C}^{p}\right)-3$. Positive values of such quantities signal entanglement. The yellow band is the error region, bounded by the extreme values $\left(\mathcal{O}_{J_{3, z}}^{\text {ap }}\left(\rho_{A B C}^{p}\right) \pm \Delta \mathcal{O}_{J_{3, z}}^{\text {ap }}\left(\rho_{A B C}^{p}\right)\right)-3$. The $J_{3, x(y)}$ cases are not reported as trivially useless, see Table 2; (b) Witnessing genuine tripartite entanglement (it is the case $N=3, k=2$ of Equation (6)). The blue dotted line depicts $\mathcal{F}_{J_{3, z}}\left(\rho_{A B C}^{p}\right)-5$, the red dashed line is $\mathcal{O}_{J_{3, z}}\left(\rho_{A B C}^{p}\right)-5$, while the red continuous line is $O_{J_{3}}^{\text {ap }}\left(\rho_{A B C}^{p}\right)-5$. The error region (yellow) is bounded by the extreme values of $\left(\mathcal{O}_{J_{3, z}}^{\mathrm{ap}}\left(\rho_{A B C}^{p}\right) \pm \Delta \mathcal{O}_{J_{3, z}}^{\mathrm{ap}}\left(\rho_{A B C}^{p}\right)\right)-5$; (c) Witnessing entanglement by computing the average values of the quantum Fisher information and the lower bound over a spin basis $\{x, y, z\}$. The blue dotted line is $\overline{\mathcal{F}}\left(\rho_{A B C}^{p}\right)-2$, the red dashed line is $\overline{\mathcal{O}}\left(\rho_{A B C}^{p}\right)-2$, while the red continuous line is $\overline{\mathcal{O}}_{J_{3}}^{\text {ap }}\left(\rho_{A B C}^{p}\right)-2$.

The yellow error region is bounded by $\left(\overline{\mathcal{O}}^{\mathrm{ap}}\left(\rho_{A B C}^{p}\right) \pm \sqrt{\sum_{i} \Delta \mathcal{O}_{J_{3, i}}^{\mathrm{ap} 2}\left(\rho_{A B C}^{p}\right)+2 \sum_{i j} \Delta \mathcal{O}_{J_{3, i}}^{\mathrm{ap}} \Delta \mathcal{O}_{J_{3, i}}^{\mathrm{ap}}}\right)-5$.

\section{Conclusions}

In this work, we provided an experimental recipe to witness multipartite entanglement by detecting asymmetry with respect to an additive Hamiltonian. We employed an experimentally friendly lower bound of the quantum Fisher information to quantify asymmetry, a geometric property of quantum systems underpinned by coherence in an observable eigenbasis. The scheme is suitable for detection of asymmetry in large scale quantum registers, as it requires a limited number of measurements regardless of the dimension of the system. We showed that in multipartite states the asymmetry lower bound with respect to additive observables is a witness of multipartite entanglement. The quantitative advantage of our method depends on several factors. We here implicitly assumed both overlap measurements and tomography to be ideal experiments where the measurements are repeated infinite times. That is, the average values obtained for both overlaps and density matrix entries match the theoretical expectation values. It is thus interesting to determine how the quantitative advantage of our method scales with the finite number of measurement repetitions which are necessary to reach an arbitrary degree of precision in estimating asymmetry. Also, the precision in determining the expectation value of the swap operator scales with the inverse of the swap value itself [62]. This suggests that for small values of the swap and a fixed degree of precision, the advantage of our method may be reduced. On the other hand, reducing exponentially the number of required measurements decreases the exposure to error sources in each detection run. Hence, a full-fledged comparison will inherently depend on the specific experimental setting (ion traps, linear optics, etc) as well as the experimental strategy (e.g., how multi-photon states are generated).

Our results pave the way for further lines of investigation. The lower bound $\mathcal{O}_{H}$ is the first faithful experimental quantifier of asymmetry for finite-dimensional systems (a second one appeared in [63]). Thus, on the experimental side, we call for a demonstration of our study. Moreover, we observe that a quadratic $\left(O\left(N^{2}\right)\right)$ sensitivity to phase shifts generated by additive Hamiltonian in $N$-party systems, 
as measured by the quantum Fisher information, has been associated to another elusive quantum effect, i.e., quantum macroscopicity [64-66]. It is clear that high values of coherence are essential to quantum macroscopicity, yet the interplay between the two concepts still needs to be clarified.

Acknowledgments: We thank Hang Li and Geza Tóth for fruitful discussions. The work was supported by the EPSRC (UK), Grant EP/L01405X/1, and the Wolfson College, University of Oxford.

Author Contributions: D. G. and B. Y. conceived the research and derived the technical results; D. G. wrote the paper.

Conflicts of Interest: The authors declare no conflict of interest.

\section{References}

1. Horodecki, M.; Oppenheim, J. (Quantumness in the context of) resource theories. Int. J. Mod. Phys. B 2013, $27,1345019$.

2. Bartlett, S.D.; Rudolph, T.; Spekkens, R.W. Reference frames, superselection rules, and quantum information. Rev. Mod. Phys. 2007, 79, 555-609.

3. Gour, G.; Spekkens, R.W. The resource theory of quantum reference frames: Manipulations and monotones. New J. Phys. 2008, 10, 033023.

4. Marvian, I. Symmetry, Asymmetry and Quantum Information. Ph.D. Thesis, University of Waterloo, Waterloo, ON, Canada, 2012.

5. Ahmadi, M.; Jennings, D.; Rudolph, T. The WAY theorem and the quantum resource theory of asymmetry. New J. Phys. 2013, 15, 013057.

6. Girolami, D.; Tufarelli, T.; Adesso, G. Characterizing Nonclassical Correlations via Local Quantum Uncertainty. Phys. Rev. Lett. 2013, 110, 240402.

7. Marvian, I.; Spekkens, R.W. Extending Noether's theorem by quantifying the asymmetry of quantum states. Nat. Commun. 2014, 5, 3821.

8. Girolami, D. Observable Measure of Quantum Coherence in Finite Dimensional Systems. Phys. Rev. Lett. 2014, 113, 170401.

9. Girolami, D.; Souza, A.M.; Giovannetti, V.; Tufarelli, T.; Filgueiras, J.G.; Sarthour, R.S.; Soares-Pinto, D.O.; Oliveira, I.S.; Adesso, G. Quantum discord determines the interferometric power of quantum states. Phys. Rev. Lett. 2014, 112, 210401.

10. Luo, S. Wigner-Yanase Skew Information and Uncertainty Relations. Phys. Rev. Lett. 2003, 91, 180403.

11. Aberg, J. Catalytic Coherence. Phys. Rev. Lett. 2014, 113, 150402.

12. Luo, S. Quantum versus classical uncertainty. Theor. Math. Phys. 2005, 143, 681-688.

13. Luo, S.; Fu, S.; Oh, C.H. Quantifying correlations via the Wigner-Yanase skew information. Phys. Rev. A 2012, 85, 032117.

14. Herbut, F. A quantum measure of coherence and incompatibility. J. Phys. A 2005, 38, 2959-2974.

15. Baumgratz, T.; Cramer, M.; Plenio, M.B. Quantifying Coherence. Phys. Rev. Lett. 2014, 113, 140401.

16. Yao, Y.; Xiao, X.; Ge, L.; Sun, C.P. Quantum coherence in multipartite systems. Phys. Rev. A 2015, $92,022112$.

17. Du, S.; Bai, Z.; Guo, Y. Conditions for coherence transformations under incoherent operations. Phys. Rev. A 2015, 91, 052120.

18. Streltsov, A.; Singh, U.; Dhar, H.S.; Bera, M.N.; Adesso, G. Measuring Quantum Coherence with Entanglement. Phys. Rev. Lett. 2015, 115, 020403.

19. Xi, Z.; Li, Y.; Fan, H. Quantum coherence and correlations in quantum system. Sci. Rep. 2015, 5, 10922.

20. Li, C.-M.; Lambert, N.; Chen, Y.-N.; Chen, G.-Y.; Nori, F. Witnessing Quantum Coherence: From solid-state to biological systems. Sci. Rep. 2012, 2, 885.

21. Monras, A.; Checinska, A.; Ekert, A.K. Witnessing quantum coherence in the presence of noise. New J. Phys. 2014, 16, 063041.

22. Pollock, F.A.; Checinska, A.; Pascazio, S.; Modi, K. Using a biased qubit to probe complex systems. Phys. Rev. A 2016, 94, 032112.

23. Altafini, C. Tensor of coherences parametrization of multiqubit density operators for entanglement characterization. Phys. Rev. A 2004, 69, 012311.

24. Bromley, T.R.; Cianciaruso, M.; Adesso, G. Frozen quantum coherence. Phys. Rev. Lett. 2015, 114, 210401. 
25. Horodecki, R.; Horodecki, P.; Horodecki, M.; Horodecki, K. Quantum Entanglement. Rev. Mod. Phys. 2009, 81, 865-942.

26. Jozsa, R.; Linden, N. On the role of entanglement in quantum-computational speed-up. Proc. R. Soc. A 2003, 459, doi:10.1098/rspa.2002.1097.

27. Giovannetti, V.; Lloyd, S.; Maccone, L. Advances in Quantum Metrology. Nat. Photonics 2011, 5, $222-229$.

28. Shimizu, A.; Morimae, T. Detection of Macroscopic Entanglement by Correlation of Local Observables. Phys. Rev. Lett. 2005, 95, 090401.

29. Huber, M.; Mintert, F.; Gabriel, A.; Hiesmayer, B.C. Detection of High-Dimensional Genuine Multipartite Entanglement of Mixed States. Phys. Rev. Lett. 2010, 104, 210501.

30. Gühne, O.; Tóth, G. Entanglement detection. Phys. Rep. 2009, 474, 1-75.

31. Helstrom, C.W. Quantum Detection and Estimation Theory; Academic Press: New York, NY, USA, 1976.

32. Tóth, G.; Apellaniz, I. Quantum metrology from a quantum information science perspective. J. Phys. A Math. Theor. 2014, 47, 424006.

33. Zhang, C.; Yadin, B.; Hou, Z.; Cao, H.; Liu, B.; Huang, Y.; Maity, R.; Vedral, V.; Li, C.; Guo, G.; et al. Determining the speed of multipartite quantum systems by few local measurements. arXiv 2016, arXiv:1611.02004.

34. Paz, J.P.; Roncaglia, A. Quantum gate arrays can be programmed to evaluate the expectation value of any operator. Phys. Rev. A 2003, 68, 052316.

35. Brun, T.A. Measuring polynomial functions of states. Quant. Inf. Comp. 2004, 4, 401-408.

36. D’Ariano, G.M.; Perinotti, P. Efficient Universal Programmable Quantum Measurements. Phys. Rev. Lett. 2005, 94, 090401.

37. Ekert, A.K.; Moura Alves, C.; Oi, D.K.L.; Horodecki, M.; Horodecki, P.; Kwek, L.C. Direct Estimations of Linear and Nonlinear Functionals of a Quantum State. Phys. Rev. Lett. 2002, 88, 217901.

38. Filip, R. Overlap and entanglement-witness measurements. Phys. Rev. A 2002, 65, 062320.

39. Jeong, H.; Noh, C.; Bae, S.; Angelakis, D.G.; Ralph, T.C. Detecting the degree of macroscopic quantumness using an overlap measurement. J. Opt. Soc. Am. B 2014, 31, 3057-3066.

40. Nakazato, H.; Tanaka, T.; Yuasa, K.; Florio, G.; Pascazio, S. Measurement scheme for purity based on two two-body gates. Phys. Rev. A 2012, 85, 042316.

41. Mintert, F.; Buchleitner, A. Observable Entanglement Measure for Mixed Quantum States. Phys. Rev. Lett. 2007, 98, 140505.

42. Walborn, S.P.; Souto Ribeiro, P.H.; Davidovich, L.; Mintert, F.; Buchleitner, A. Experimental determination of entanglement with a single measurement. Nature 2006, 440, 1022-1024.

43. Oszmaniec, M.; Kuś, M. Universal framework for entanglement detection. Phys. Rev. A 2013, 88, 052328.

44. Jin, J.; Zhang, F.; Yu, C.; Song, H. Direct scheme for measuring the geometric quantum discord. J. Phys. A Math. Theor. 2012, 45, 115308.

45. Yu, S. Quantum Fisher Information as the Convex Roof of Variance. arXiv 2013, arXiv:1302.5311.

46. Tóth, G.; Petz, D. Extremal properties of the variance and the quantum Fisher information. Phys. Rev. A 2013, 87, 032324.

47. Petz, D. Monotone metrics on matrix spaces. Linear Algebra Its Appl. 1996, 244, 81-96.

48. Gibilisco, P.; Imparato, D.; Isola, T. Inequalities for quantum Fisher information. Proc. Am. Math. Soc. 2008, 137, 317-327.

49. Paiva Pires, D.; Céleri, L.C.; Soares-Pinto, D.O. Geometric lower bound for a quantum coherence measure. Phys. Rev. A 2015, 91, 042330.

50. Wang, X.; Schirmer, S.G. Contractivity of the Hilbert-Schmidt distance under open-system dynamics. Phys. Rev. A 2009, 79, 052326.

51. Tufarelli, T.; Girolami, D.; Vasile, R.; Bose, S.; Adesso, G. Quantum resources for hybrid communication via qubit-oscillator states. Phys. Rev. A 2012, 86, 052326.

52. Piani, M. Problem with geometric discord. Phys. Rev. A 2012, 86, 034101.

53. Girolami, D.; Vasile, R.; Adesso, G. Theoretical insights on measuring quantum correlations. Int. J. Mod. Phys. B 2013, 27, 1345020.

54. Pezzé, L.; Smerzi, A. Ultrasensitive Two-Mode Interferometry with Single-Mode Number Squeezing. Phys. Rev. Lett. 2013, 110, 163604.

55. Tóth, G. Multipartite entanglement and high-precision metrology. Phys. Rev. A 2012, 85, 022322. 
56. Hyllus, P.; Laskowski, W.; Krischek, R.; Schwemmer, C.; Wieczorek, W.; Weinfurter, H.; Pezzé, L.; Smerzi, A. Fisher information and multiparticle entanglement. Phys. Rev. A 2012, 85, 022321.

57. Hong, Y.; Luo, S.; Song, H. Detecting k-nonseparability via quantum Fisher information. Phys. Rev. A 2015, 91, 042313.

58. Li, N.; Luo, S. Entanglement detection via quantum Fisher information. Phys. Rev. A 2013, 88, 014301.

59. Luo, S. Wigner-Yanase skew information vs. quantum Fisher information. Proc. Am. Math. Soc. 2003, 132, 885-890.

60. Gühne, O.; Seevinck, M. Separability criteria for genuine multiparticle entanglement. New J. Phys. 2010, 12, 053002.

61. Apellaniz, I.; Lücke, B.; Peise, J.; Klempt, C.; Tóth, G. Detecting metrologically useful entanglement in the vicinity of Dicke states. New J. Phys. 2015, 17, 083027.

62. Daley, A.J.; Pichler, H.; Schachenmayer, J.; Zoller, P. Measuring Entanglement Growth in Quench Dynamics of Bosons in an Optical Lattice. Phys. Rev. Lett. 2012, 109, 020505.

63. Marvian, I.; Spekkens, R.W. How to quantify coherence: Distinguishing speakable and unspeakable notions. Phys. Rev. A 2016, 94, 052324.

64. Leggett, A. Macroscopic Quantum Systems and the Quantum Theory of Measurement. Prog. Theor. Phys. Supp. 1980, 69, 80-100.

65. Fröwis, F.; Dür, W. Measures of macroscopicity for quantum spin systems. New J. Phys. 2012, 14, 093039.

66. Yadin, B.; Vedral, V. A general framework for quantum macroscopicity in terms of coherence. Phys. Rev. A 2016, 93, 022122.

(C) 2017 by the authors. Licensee MDPI, Basel, Switzerland. This article is an open access article distributed under the terms and conditions of the Creative Commons Attribution (CC BY) license (http://creativecommons.org/licenses/by/4.0/). 